\title{
Speed: Uma solução inter-veicular para detectar e controlar vias congestionadas no TMS
}

\author{
Geraldo P. Rocha Filho ${ }^{1}$, Rodolfo I. Meneguette ${ }^{2}$, Gustavo Pessin ${ }^{3}$, \\ Jó Ueyama ${ }^{4}$, Leandro A. Villas ${ }^{5}$ \\ ${ }^{1}$ Departamento de Ciência da Computação, Universidade de Brasília - UnB \\ ${ }^{2}$ Instituto Federal de São Paulo - IFSP \\ ${ }^{3}$ Instituto Tecnológico Vale - ITV \\ ${ }^{4}$ Instituto de Ciência Matemáticas e de Computação, Universidade de São Paulo - USP \\ ${ }^{5}$ Instituto de Computação - Universidade Estadual de Campinas - UNICAMP \\ geraldofeunb.br, meneguettedifsp.edu.br, gustavo.pessinditv.org \\ joueyama@icmc.usp.br, leandrodic.unicamp.br
}

\begin{abstract}
This work proposes a solution with inter-vehicular communication to estimate and maximize the congestion in TMS, named Speed. For this, Speed is modeled based on an ensemble of classifiers to increase reliability when classifying vehicle traffic. Based on a communication mechanism, such classification is used to carry out the dissemination of information. When compared with other works of the literature, Speed showed to advance in the state of the art by having an increase in the precision of congestion levels classification while reducing: (i) the emission of $\mathrm{CO}_{2}$ by 3.37\%; (ii) fuel consumption by 3\%; and (iii) travel time by 1.02 times faster than the other works.
\end{abstract}

Resumo. Este artigo propõe uma solução com comunicação inter-veicular para estimar e maximizar o congestionamento no TMS, nomeado Speed. Para isso, o Speed é modelado com base em um comitê de classificadores que tem como objetivo aumentar a confiabilidade no momento da classificação do tráfego de veículos. Com base em um mecanismo de comunicação, tal classificação é utilizada para realizar a disseminação da informação. Ao comparar o Speed com outros trabalhos da literatura, a nossa solução mostrou avançar o estado da arte por possuir um aumento nos acertos dos niveis de congestionamento ao mesmo tempo que reduz: (i) a emissão de $\mathrm{CO}_{2}$ em 3,37\%; (ii) o consumo de combustível em 3\%; e (iii) o tempo de viagem em 1.02 vezes.

\section{Introdução}

Atualmente, observa-se um rápido aumento do número de veículos que trafegam nas grandes metrópoles. Esse aumento, atribuído ao crescimento populacional e aos cidadãos que usam seus próprios veículos como meio de locomoção [Junior et al. 2015], ocasiona diversos problemas no sistema de transporte, sendo o congestionamento um dos fatores a ser destacado. Ainda por causa desse crescimento, também surgem outros problemas que atingem o trânsito [Meneguette et al. 2018], como o desvio de tráfego e 
a falta de segurança dos condutores devido a um evento na via urbana, por exemplo. Tais problemas atingem gravemente a economia quando vista como um todo. No Brasil, o custo estimado do congestionamento nas cidades ultrapassa os $\mathrm{R} \$ 80$ bilhões por ano [Cintra 2013]. Já na União Européia é de aproximadamente 2\% do seu Produto Interno Bruto (PIB) [H. Allen and Stonehill 2013] e nos EUA o custo é de mais de US\$ 160 bilhões [Texas Transportation Institute 2015].

Salienta-se que o problema de congestionamento nas cidades não é uma tarefa trivial de ser resolvida. Apesar da ampliação da rede de transporte auxiliar no problema do congestionamento, essa expansão pode ser inviável devido ao elevado custo financeiro, às restrições ambientais e geográficas impostas, bem como ao tempo elevado para finalizar a melhoria da infraestrutura de transporte. Tais limitações podem ser minimizadas pelo Sistema de Gerenciamento de Tráfego [Souza et al. 2017, Meneguette et al. 2018, Cunha et al. 2018] (TMS, do inglês Traffic Management Systems) que surge como uma alternativa promissora para auxiliar no problema do congestionamento nas cidades. O TMS pode ser definido como um tipo especial de serviço das Redes Ad-Hoc Veiculares (VANETs, do inglês Veicular Ad-Hoc Networks) munido com tecnologias de comunicação, detecção e processamento para coletar dados do tráfego com o intuito de lidar com a sua melhoria, não apenas em relação a determinados veículos, mas também no que tange todo o sistema de transporte da cidade.

Diferentes soluções foram propostas para lidar com o problema do congestionamento de tráfego nas cidades. Algumas soluções sugerem as melhores rotas individuais para evitar o congestionamento [Doolan and Muntean 2013, Doolan and Muntean 2017], o que pode gerar novos congestionamentos em outras áreas. Outras soluções têm se limitado em detectar congestionamento [Wongcharoen and Senivongse 2016, Nottle et al. 2017] ou redirecionar veículos [Pan et al. 2012, Pan et al. 2017] ou propostas para cenários específicos [Meneguette et al. 2015, Severino et al. 2018] como urbano ou rodoviário, resolvendo apenas uma parte do problema. Ainda há trabalhos que modelam algoritmos de aprendizagem de máquina para estimar o congestionamento da via [Araujo et al. 2014, Souza et al. 2015, Meneguette et al. 2016]. Entretanto, tal modelagem deve lidar com uma grande quantidade de dados obtidos de maneira implícita providos do TMS para monitorar o tráfego.

Ainda que haja um esforço crescente conquistado no TMS, aumentar a precisão de acertos das vias congestionadas para maximizar o fluxo de veículos na infraestrutura de transporte, traz uma nova questão de pesquisa, a saber: Como prover uma maior precisão para estimar o nível de congestionamento da via, maximizando o fluxo de veículos na infraestrutura de transporte? Tal questão é uma nova problemática que está sendo abordada na literatura e que este artigo investiga.

Este trabalho apresenta o Speed, uma solução inter-veicular para estimar o congestionamento e maximizar o fluxo de tráfego de veículos no sistema de transporte. O Speed é baseado em um comitê de classificadores que tem como objetivo aumentar a confiabilidade no momento da classificação do tráfego de veículos. Com isso, tal classificação é utilizada para realizar a disseminação da informação com base em um módulo de comunicação Publish/Subscribe que, além de encaminhar as mensagens para os grupos de interesse, evita o problema de broadcast storms. Para certificar a eficiência do Speed com relação ao problema de congestionamento, a solução proposta foi com- 
parada com outras soluções consagradas na literatura que mais assemelham a esta pesquisa [Araujo et al. 2014, Souza et al. 2015, Meneguette et al. 2016]. Os resultados obtidos por meio de simulações, evidenciam um aumento nos acertos dos níveis de congestionamento com redução no tempo de viagem dos condutores, no consumo de combustível dos veículos e na emissão de $\mathrm{CO}_{2}$.

O restante deste artigo está organizado da seguinte forma. Na Seção 2, são apresentados os principais trabalhos relacionados a detecção de congestionamento de veículos no TMS. A Seção 3 apresenta a modelagem do Speed, enquanto que sua avaliação de desempenho com os resultados obtidos são descritos na Seção 4. Por fim, na Seção 5, são apresentadas as conclusões e trabalhos futuros.

\section{Trabalhos relacionados}

Diversas soluções foram propostas para lidar com o problema de congestionamento nos últimos anos. Além de trazer maior conforto e segurança para os condutores [Pessin et al. 2014, Meneguette et al. 2018], as soluções propostas tem como objetivo otimizar o tráfego de veículos no sistema de transporte, reduzindo o tempo de viagem, o consumo de combustível e a quantidade de $\mathrm{CO}_{2}$ emitidos na atmosfera. Entretanto, modelar um mecanismo para estimar o nível de congestionamento da via com um aumento nos acertos das vias congestionadas, e com um tempo aceitável para alertar os condutores não é uma tarefa trivial.

As soluções apresentadas em [Bauza et al. 2010, Bauza and Gozálvez 2013, Araujo et al. 2014], propõem serviços de gerenciamento de tráfego para detectar o congestionamento da via com base na lógica fuzzy. Baseado nos trabalhos [Bauza et al. 2010, Bauza and Gozálvez 2013], os autores [Araujo et al. 2014] propõem o CARTIM, um protocolo para detectar e reduzir o congestionamento de veículos no sistema de transporte. Para isso, o CARTIM utiliza como entrada para a lógica fuzzy a velocidade e a densidade dos veículos na via para classificar o nível de congestionamento localmente, disseminando-o de maneira cooperativa entre os veículos. Em seguida, caso a via esteja congestionada, o CARTIM adota uma heurística para alterar as rotas dos veículos. Embora o tempo de inferência da lógica fuzzy seja rápido o suficiente para alertar os motoristas, a sua precisão para detectar o nível de congestionamento depende dos valores de entrada. Ainda, como os veículos não possuem conhecimento global da condição do tráfego, novas áreas podem ficar congestionadas, ou a heurística de sugestão de rotas pode indicar rotas inapropriadas.

Em [Souza et al. 2015], é proposto o CO-OP, uma solução para prevenção e controle de congestionamentos com base em um roteamento cooperativo. O CO-OP modela um mecanismo de classificação para detectar diferentes níveis de congestionamento na via utilizando K-Nearest Neighbor (KNN). Para isso, a densidade da rua combinada com a velocidade média da via é utilizada como parâmetro de entrada para classificar a condição do tráfego. A informação da classificação é disseminada para os veículos que estão dentro de uma área de roteamento. Tal área, é utilizada para evitar roteamentos desnecessários para outros veículos. Com isso, apenas os veículos que possuem maior probabilidade de congestionamento recebem a informação. O KNN apresenta um tempo rápido de inferência devido a sua baixa complexidade quando comparado com a lógica fuzzy. Entretanto, quando comparado com os demais mecanismos de classificação, o 
KNN possui menor precisão para detectar a condição do tráfego, como constatado nesta pesquisa.

Outra solução que se assemelha com esta pesquisa é apresentada em [Meneguette et al. 2016]. Os autores [Meneguette et al. 2016] propõem o INCIDEnt, um protocolo de detecção, disseminação e controle de congestionamento para ambientes urbanos e rodoviários. Diferente do CARTIM e CO-OP, o INCIDEnt é baseado em uma Rede Neural Artificial do tipo MultiLayer Perceptron (RNA-MLP), a qual é modelada para detectar e classificar os níveis de congestionamentos nas vias. Ainda, a classificação por meio da RNA-MLP é utilizada para realizar a disseminação da informação na rede, além de ser aplicada no mecanismo de sugestão de novas rotas para evitar que os motoristas passem em vias congestionadas. Embora tal mecanismo de classificação permita um auto aprendizado, a configuração pode afetar no desempenho do sistema. Com isso, a maneira como a RNA-MLP é modelada poderá impactar no tempo de decisão que é levado em conta para realizar a sugestão da rota, bem como na precisão da classificação da condição do tráfego.

Diferentemente dos trabalhos da literatura que apresentam um único método de classificação, dos quais a precisão para detectar o congestionamento dependerá da configuração do método, neste trabalho será modelado um comitê de classificadores que busca maximizar a precisão na classificação sem impactar o tempo de decisão da rota. O uso do comitê visa o aperfeiçoamento da taxa de acerto da classificação, além de permitir uma maior adaptação de novos eventos e condições que possam ocorrer. Ainda, a solução proposta permite a classificação dos níveis de congestionamento de tráfego de veículos sem uma sobrecarga de informações de controle na rede, evitando assim colisões de informações de controle, dando maior vazão nas informações. Salienta-se que o mecanismo de classificação proposto é uma ferramenta fundamental que é utilizada para a realização da disseminação da informação na rede.

\section{Uma Solução inter-veicular para detectar e controlar vias congestionadas}

Esta seção apresenta o Speed, uma solução inter-veicular para estimar o congestionamento e maximizar o fluxo de tráfegos de veículos nas vias. O Speed é composto por um comitê de classificadores para classificar e detectar o nível de congestionamento localmente, além de aumentar a precisão de acertos das vias congestionadas. Ainda, implementou-se no Speed um módulo de comunicação Publish/Subscribe (Pub/Sub) capaz de disseminar o conteúdo de acordo com o nível de congestionamento da via, o que evita o problema de broadcast storms. Além disso, um mecanismo de planejamento de novas rotas para maximizar o fluxo de tráfegos da via foi modelado no Speed. O Speed tem como objetivo principal prover maior precisão para estimar o nível de congestionamento da via, tendo como meta específica maximizar o fluxo de tráfego dos veículos no sistema de transporte.

No Speed, assume-se que cada veículo é equipado com uma On-Board Unit (OBU) para obter sua velocidade e localização. A Figura 1 sumariza o funcionamento do Speed, o qual detecta o congestionamento e em seguida realiza a troca de rota para maximizar o fluxo de tráfego no sistema de transporte. Para tanto, o veículo que detectou o congestionamento (Rótulo 1, Figura 1), inicia uma disseminação de aviso de congestionamento para notificar os motoristas. Tal aviso chega para o motorista distante (Rótulo 


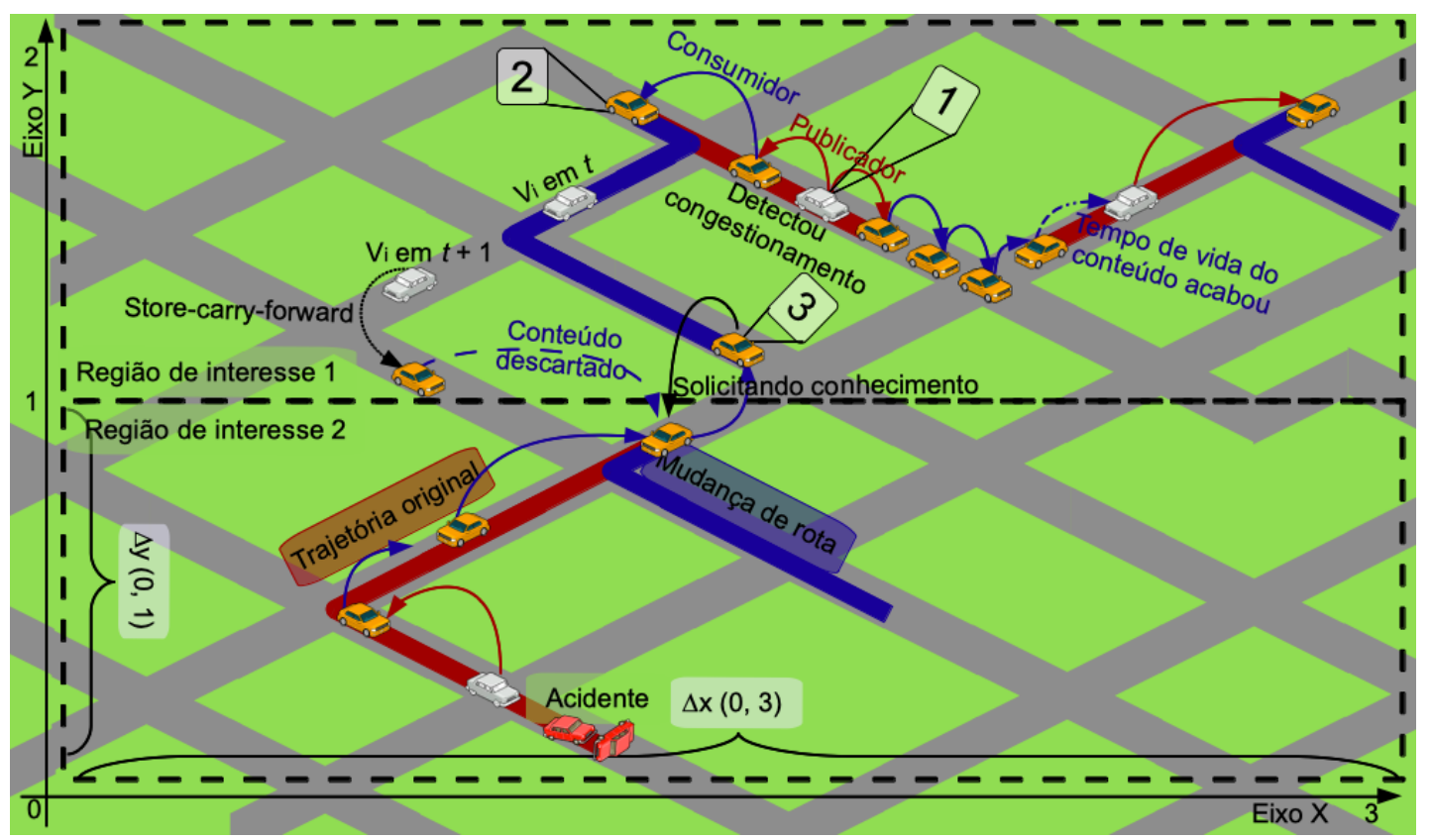

Figura 1. Funcionamento da solução inter-veicular para maximizar o fluxo de tráfegos no sistema de transporte.

2, Figura 1) que ainda não entrou na via congestionada. Com isso, é possível recalcular um novo plano de rota (Rótulo 3, Figura 1) para os veículos evitarem a via congestionada. Para um melhor entendimento do Speed, a solução proposta é detalhada em quatro etapas: (i) modelo de dados para estimar o congestionamento; (ii) mecanismo para detectar congestionamento; (iii) mecanismo para disseminar a informação do congestionamento; e (iv) mecanismo de recomendação de rotas.

\subsection{Modelo de dados para estimar o congestionamento}

Estimar o nível de congestionamento em uma via não é processo trivial [Bauza et al. 2010, Rao and Rao 2012], uma vez que é necessário correlacionar e lidar com métricas qualitativas (acidentes, infraestrutura da via e eventos especiais como reuniões sociais em massa) e quantitativas (velocidade e tempo de viagem). Tal estimativa ainda é mais problemática, quando se trata de dados obtidos de maneira implícita providos do TMS. Para este trabalho, foi adotado o modelo de classificação estendido do Skyscomp [SKYCOMP 2008], o qual foi aplicado em [Bauza et al. 2010, Araujo et al. 2014, Souza et al. 2015, Meneguette et al. 2016]. O Skyscomp fornece um Level-Of-Service $F$ (LOS), o qual representa uma métrica para classificar o fluxo de tráfego de uma via, definida pelo Highway Capacity Manual (HCM) [Board 2000].

O modelo de classificação adotado na $\operatorname{LOS} F$, considera como atributo a velocidade do veículo e a densidade de veículos na via para fornecer o nível do congestionamento do tráfego, como apresentado na Tabela 1. Nesse caso, foram estabelecidos três níveis de congestionamento, sendo eles: (i) livre; (ii) moderado; e (iii) congestionado. Fundamentado em [Meneguette et al. 2016], o Speed também obtém a velocidade do veículo por meio da OBU. Já a densidade do veículo é obtida pela quantidade de 
veículos vizinhos que estão em sua área de cobertura. A seguir, é apresentado o mecanismo para detectar o nível de congestionamento da via.

Tabela 1. Relação entre a densidade da via e velocidade do veículo para estimar o nível de congestionamento [Bauza et al. 2010].

\begin{tabular}{|l|l|l|}
\hline Nível de congestionamento & densidade & velocidade \\
\hline Livre & {$[29-37] \mathrm{vel} / \mathrm{km} / \mathrm{ln}$} & {$[48-81] \mathrm{km} / \mathrm{h}$} \\
\hline Moderado & {$[37-50] \mathrm{vel} / \mathrm{km} / \mathrm{ln}$} & {$[24-64] \mathrm{km} / \mathrm{h}$} \\
\hline Congestionado & $>=50 \mathrm{vel} / \mathrm{km} / \mathrm{ln}$ & {$[0-40] \mathrm{km} / \mathrm{h}$} \\
\hline
\end{tabular}

\subsection{Mecanismo para detectar congestionamento}

O mecanismo de detecção tem por objetivo estimar o nível de congestionamento da via. Há diversos trabalhos que utilizam algoritmos de classificação para detectar o nível de congestionamento. Particulamente em [Araujo et al. 2014, Souza et al. 2015, Meneguette et al. 2016], são utilizados a lógica fuzzy, o K-Nearest Neighbors (KNN) e a Rede Neural Artificial do tipo MultiLayer Perceptron (RNA-MLP), respectivamente. Entretanto, o uso de classificadores individuais além de depender de ajustes nos parâmetros para a classificação, possuem erros característicos que podem resultar em classificações errôneas, dependendo do problema ou do dataset utilizado [Canuto 2001, Santos 2008].

Para tratar tal limitação, o mecanismo proposto para estimar o nível de congestionamento nas vias no Speed foi modelado por meio de um comitê de classificadores. Para facilitar o entendimento do comitê no Speed, a Figura 2 apresenta o seu fluxo de funcionamento. O comitê foi modelado com base nos algoritmos de classificação adotados em [Araujo et al. 2014, Souza et al. 2015, Meneguette et al. 2016], sendo eles, respectivamente: (i) Lógica fuzzy; (ii) KNN; e (iv) RNA-MLP. O comitê utiliza como atributo de entrada a velocidade do veículo e a densidade de veículos vizinhos e, mediante uma agregação de respostas dos algoritmos de classificação, estima o nível de congestionamento da via. A agregação da resposta ocorre por meio de um critério de votação dado pela formula: $\bar{W} x_{i}=\frac{A P x_{i}}{\sum_{j}^{n} A P x_{j}}$, onde $\bar{W}$ são os pesos atribuídos para cada algoritmo $x$ de acordo com sua taxa de precisão $A P$. Portanto, o comitê considera o mérito de cada $x$ para aumentar a precisão nos acertos em estimar o nível de congestionamento da via, uma vez que não é persuadido por decisões errôneas de apenas um algoritmo.

A resposta final do comitê foi normalizada entre 0 e 1 . Com isso, foram estabelecidos três níveis de congestionamento: (i) livre, entre o intervalo [0, $\left.\frac{1}{3}\right]$; (ii) moderado, entre o intervalo $] \frac{1}{3}, \frac{2}{3}\left[\right.$; e (iii) congestionado, entre o intervalo $\left[\frac{2}{3}, 1\right]$. Empiricamente, tal divisão possui uma convergência mais rápida para estimar o nível do congestionamento. A seguir, é apresentado o mecanismo para disseminar a informação que foi detectada na via.

\subsection{Mecanismo para disseminar dados}

Após detectar o nível de congestionamento, o Speed dissemina a informação entre os veículos. Vale salientar que as informações são entregues para múltiplos veículos que são consumidores de informações e não há conexões diretas com os produtores de informações (isto é, veículo que detectou o congestionamento). Por isso, o mecanismo 


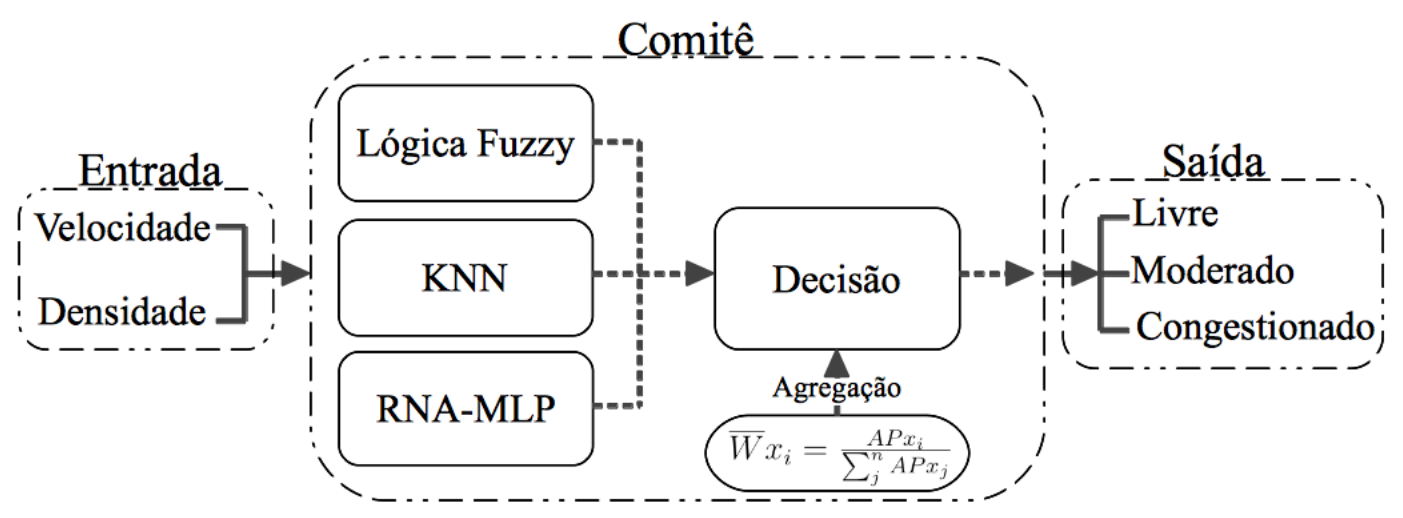

Figura 2. Funcionamento do comitê de classificadores modelado para estimar o nível de congestionamento.

de disseminação do Speed é modelado com base no paradigma Pub/Sub. O Pub/Sub permite a disseminação das informações entre os veículos de maneira assíncrona. Ainda, o Pub/Sub permite que a conexão entre os veículos seja fracamente acoplada, o que não traz dano em uma topologia dinâmica como as das TMSs, uma vez que tais veículos podem perder a conexão por diversos motivos.

Antes de iniciar o processo de publicação, assume-se que o mapa é dividido em regiões de interesse (RI), sendo que cada RI é independente uma da outra. Isso irá evitar o problema da broadcast storm. Entende-se por RI, como uma parte do mapa em que a informação relevante é propagada aos veículos que necessitam dela. Com isso, cada veículo tem conhecimento da sua RI (por exemplo, nível de congestionamento ou acidente). Para adquirir o conhecimento de um outra RI, um veículo pode tentar compartilhar dados com outros veículos que estão nas outras RIs por meio de um modelo request-reply via Pub/Sub. O RI pode ser ajustável dependendo da característica do ambiente, por exemplo, em um horário de pico em uma determinada via o RI pode ser menor para garantir a escalabilidade das TMSs. Entretanto, para esta solução, considerou-se o tamanho do mapa e as coordenadas para formar as RIs (Figura 1). Por exemplo, em um mapa de $2 \mathrm{~km}$ por $3 \mathrm{~km}$, uma RI seria formada pelas coordenadas cartesianas $\Delta x_{i}$ e $\Delta y_{i}$ com deslocamento no eixo $x(0,3)$ e eixo $y(0,1)$. Por meio da OBU os veículos conseguem localizar-se, e consequentemente saberem em quais RIs do mapa eles estão. Para estabelecer a comunicação entre os veículos nas suas respectivas regiões, considerou-se duas funções que são implantadas nos veículos:

- Produtor de informações. Coleta os dados do tráfego, em seguida detecta o nível de congestionamento, disseminando-o para os veículos consumidores de informações. O primeiro veículo que detecta se a via está congestionada é considerado produtor de informação daquela região.

- Consumidor de informações. Recebe a informação, compartilhando-a para a sua RI.

O processo de disseminação ocorre quando o veículo realiza a estimação do nível do congestionamento por meio do comitê. Com isso, tal veículo torna-se produtor de informação $V_{p i}$. O $V_{p i}$ cria uma mensagem de publicação contendo o seu ID, a sua localização, o conteúdo do evento (isto é, o nível de congestionamento) e o tempo de vida 
do conteúdo. O $V_{p i}$ dissemina o conteúdo de acordo com o nível de congestionamento da via. Por exemplo, se o veículo está em uma via congestionada, além do veículo deslocar mais lentamente, não há mudanças significativas no conteúdo (por exemplo, velocidade e localização) para ser enviado. Desta forma, pode-se utilizar um tempo maior (menor) para disseminar o conteúdo quando o nível de congestionamento é alto (baixo). Com isso, é possível definir a disseminação pelo intervalo do nível de congestionamento, como definido na Seção 3.2, para evitar o problema da broadcast storm, o qual é minimizado pela divisão do mapa em RIs.

Após o $V_{p i}$ publicar o conteúdo, os seus veículos vizinhos recebem o evento publicado. Tais veículos tornam-se consumidores de informações, $V_{c i}$. Para os $V_{c i}$ receberem o conteúdo, tem-se que subescrever no barramento de evento do pub/sub, com uma mensagem contendo o ID, o conteúdo a ser recebido, a coordenada de GPS do inscrito e o caminho que o inscrito passará. Além disso, o $V_{c i}$ também se inscreve em outros veículos da região para receber dados de outros $V_{c n}$. Com isso, o $V_{c i}$ obtém a informação do conteúdo local de onde ocorreu o congestionamento e outras informações da RI. Ao receber o conteúdo, o $V_{c i}$ verifica se está na RI. Em seguida verifica se o tempo de vida do conteúdo acabou ou se o conteúdo é uma duplicata. Somente em caso afirmativo, o $V_{c i}$ descarta o conteúdo. Do contrário, o conteúdo é repassado para os veículos que estão na região de acordo com o nível de congestionamento estimado. Neste caso, pode ocorrer fragmentação da rede devido, por exemplo, a falta de conectividade entre os veículos, resultando em mensagens perdidas. Por isso, foi utilizado um mecanismo de store-carryforward que usa os beacons enviados dos veículos. O store-carry-forward armazena a informação e aproveita a mobilidade do veículo para transportá-la para diferentes partes da região. O processo para a troca de rotas é descrito a seguir.

\subsection{Mecanismo de recomendação de rotas}

Nesta seção, é apresentado o mecanismo de recomendação de rotas do Speed para evitar que os veículos passem por uma via congestionada. A recomendação ocorre quando os veículos recebem a informação que a via está congestionada por meio de um beacon. Com isso, o veículo verifica se passará pela rota congestionada. Caso positivo, o veículo realiza a troca de rota e atualiza tal informação em sua mensagem. Entretanto, se o veículo recebe a informação que o trânsito está livre ou moderado, o veículo apenas atualiza tal informação em sua mensagem e não realiza a troca de rota.

\section{Avaliação de Desempenho e Metodologia}

Esta seção apresenta os resultados da avaliação de desempenho, bem como a metodologia utilizada para gerar os resultados. Para isso, o Speed foi validado em duas etapas, a saber: (i) avaliação de desempenho para determinar a capacidade do Speed em estimar o nível de congestionamento das vias; e (ii) avaliação de desempenho da gerência de tráfego no TMS usando o Speed. Com tais avaliações, foi possível comparar o desempenho do Speed frente aos trabalhos da literatura. Os cenários modelados, as métricas utilizadas e os resultados alcançados juntamente com os parâmetros selecionados são apresentados nas próximas subseções.

\subsection{Avaliação de desempenho para estimar o nível de congestionamento}

Nesta subseção, é avaliado o desempenho do comitê que foi modelado no Speed para estimar o nível de congestionamento nas vias, comparando-o com os algoritmos 
Fuzzy, KNN e RNA-MLP que foram adotados em [Araujo et al. 2014, Souza et al. 2015, Meneguette et al. 2016], respectivamente. Para a obtenção dos modelos de classificação, utilizou-se a base de dados que foi construída com base no HCM. Após a construção da base de dados, utilizou-se a técnica $k$-fold cross-validation com $k=10$ para separar o conjunto de teste e treino. Com isso, foi usado $k$-1 subconjunto para treinamento e o restante do subconjunto para teste. De maneira rotativa, o subconjunto de treinamento é alternado $k$ vezes para fornecer uma estimativa mais confiável dos modelos de classificação construídos. Como o objetivo é avaliar o quão correto é estimado o nível de congestionamento, utilizou-se como variável de respostas as métricas: (i) precisão; (ii) recall; (iii) F-Measure; e (iv) Erro Médio Quadrático, EMQ. Tais métricas são calculadas a partir da matriz de confusão, como apresentado na Figura 3, na qual a linha representa a classificação gerada pelos algoritmos com base nas perdas causadas, e a coluna os dados de referência rotulados.

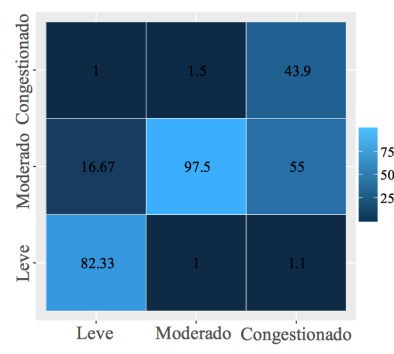

(a) Fuzzy

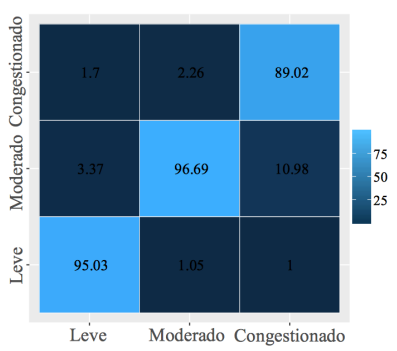

(b) $\mathrm{KNN}$

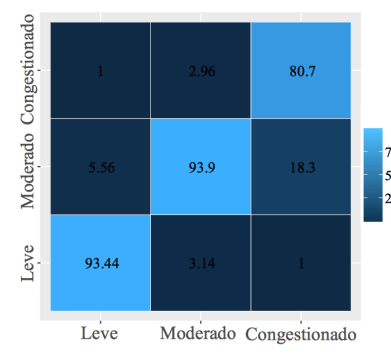

(c) RNA-MLP

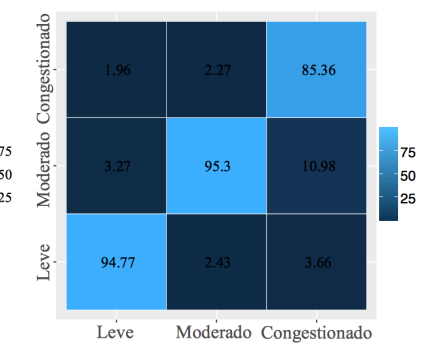

(d) Comitê

Figura 3. Matriz de confusão em porcentagem utilizada para calcular as métricas da Tabela 2.

A Tabela 2, derivada da Figura 3, sumariza os resultados das métricas em função dos algoritmos avaliados. Os melhores resultados estão destacados em negrito. Nota-se que o comitê modelado no Speed foi o que obteve os melhores valores, independente das métricas utilizadas. Em particular para a métrica precisão, o comitê foi 0.98 vezes mais preciso no pior caso e 1.06 vezes mais preciso no melhor caso. Isso faz sentido, pois o comitê modelou o problema de estimar o nível de congestionamento por meio da combinação de classificadores. Em razão disso, os erros gerados por um classificador isolado não são cometidos. Portanto, existe um ligeiro aumento nos acertos para estimar o nível de congestionamento e, consequentemente sua generalização no processo de decisão para a detecção da via congestionada. Portanto, o mecanismo proposto possui uma melhor taxa de acertos para estimar o nível de congestionamento quando comparado com os modelos de classificação individualizados.

\subsection{Avaliação de desempenho da gerência de tráfego}

Esta subseção avalia o fluxo de tráfego de veículo usando o Speed, apresentando sua eficiência no TMS. Para isso, o Speed é comparado com o INCIDEnt [Meneguette et al. 2016] e OVMT ${ }^{1}$ que é o tráfego original de mobilidade do veículo. Os experimentos foram realizados por meio de simulações utilizando o OM$\mathrm{NeT}++5.0^{2}$. Para o cenário urbano, utilizou-se um fragmento de $1 \mathrm{~km}^{2}$ da cidade de Ma-

\footnotetext{
${ }^{1}$ OVMT, do inglês Original Vehicle Mobility Traffic

${ }^{2} \mathrm{OMNeT++}$, Discrete Event Simulator, http://www.omnetpp.org
} 
Tabela 2. Avaliação dos algoritmos de classificação.

\begin{tabular}{|l|c|c|c|c|}
\hline \multirow{2}{*}{ Algoritmos } & \multicolumn{4}{|c|}{ Métricas } \\
\cline { 2 - 5 } & Precisão & Recall & F-Measure & EMQ $^{1}$ \\
\hline Fuzzy & $0,91 \pm 0,041$ & $0,92 \pm 0,043$ & 0,91 & 0,26 \\
\hline KNN & $0,89 \pm 0,038$ & $0,90 \pm 0,036$ & 0,89 & 0,21 \\
\hline RNA-MLP & $0,93 \pm 0,031$ & $0,94 \pm 0,033$ & 0,93 & 0,13 \\
\hline Comitê & $\mathbf{0 , 9 4} \pm 0,027$ & $\mathbf{0 , 9 6} \pm 0,024$ & $\mathbf{0 , 9 5}$ & $\mathbf{0 , 1 1}$ \\
\hline \multicolumn{5}{|c|}{${ }^{1}$ EMQ - Erro Médio Quadrático. }
\end{tabular}

nhattan, Nova Iorque, Estados Unidos, que foi gerado com o auxílio do SUMO 0.28.0 3 . O SUMO permite criar o modelo de mobilidade dos veículos, bem como a construção do cenário que foi extraído do OpenStreetMap ${ }^{4}$. No cenário gerado, os veículos viajam em ambas as direções ao longo da rua com uma velocidade de 0-60 km/ $\mathrm{h}$. Para prover uma condição de tráfego com vias congestionadas, a densidade da mobilidade dos veículos no cenário é de 300 veículos $/ \mathrm{km}^{2}$. Como é realístico assumir que há mais carros que ônibus e caminhões em uma via, considerou-se as seguintes quantidades de veículos: (i) $1 / 2$ para carros; (ii) 1/4 para ônibus; e (iii) 1/4 para caminhões. Para realizar a comunicação inter-veículos, utilizou-se o framework Veins $4.5^{5}$ que foi integrado no OMNeT++. Além disso, o Veins possui implementado o IEEE 802.11p e modelos de atenuação de sinal para simular obstáculos na via. A Tabela 3 apresenta o conjunto de parâmetros que foi estabelecido para realizar a simulação.

Tabela 3. Conjunto de parâmetros utilizados na simulação.

\begin{tabular}{|l||l|}
\hline \multicolumn{1}{|c||}{ Parâmetro } & Valor padrão \\
\hline \hline Cenário & Manhattan \\
Área de simulação & $1 \mathrm{~km}^{2}$ \\
Densidade & 300 veículos $/ \mathrm{km}^{2}$ \\
Potência de transmissão & $0.98 \mathrm{~mW}$ \\
Raio de transmissão & $200 \mathrm{~m}$ \\
Taxa de transmissão & $18 \mathrm{Mbit} / \mathrm{s}$ \\
Camada MAC & $802.11 \mathrm{p}$ \\
\hline
\end{tabular}

Como o objetivo da avaliação é determinar o desempenho da gerência de tráfego usando o Speed, utilizou-se as seguintes métricas: (i) tempo de viagem, representa o tempo médio de viagem gasto de todos os veículos a partir de uma origem até o destino; (ii) emissão de $\mathrm{CO}_{2}$, representa a média de $\mathrm{CO}_{2}$ emitido durante o tempo de viagem de todos os veículos; e (iii) consumo de combustível, representa a média do consumo de combustível durante o tempo de viagem de todos os veículos. Para obter tais métricas, utilizou-se o EMIT que foi integrado no SUMO. O EMIT é um modelo estatístico que é derivado do $\mathrm{HBEFA}^{6}$ que estima o tempo de viagem, as emissões de $\mathrm{CO}_{2}$ e consumo de combustível considerando a velocidade do carro e sua aceleração. As simulações fo-

\footnotetext{
${ }^{3}$ SUMO, Simulation of Urban MObility, http://sumo.dlr.de/index.html

${ }^{4}$ OpenStreetMap, http://www.openstreetmap.org/

${ }^{5}$ Veins, http://veins.car2x.org

${ }^{6} \mathrm{HBEF}$, Handbook Emission Factors for Road Transport, http://www.hbefa.net
} 
ram replicadas 33 vezes com $95 \%$ de confiança com base na distribuição $t$-student. Os resultados obtidos da simulação são apresentados a seguir.

\subsubsection{Análise dos resultados obtidos}

A Figura 4a apresenta os resultados pertencentes à métrica tempo de viagem, realizando uma análise comparativa do Speed com o INCIDEnt e o OVMT. Nota-se que o Speed possui um tempo de viagem menor independente das soluções, INCIDEnt e OVMT. No pior caso que é usando o INCIDEnt, o Speed realiza o tempo de viagem em média 1.02 vezes mais rápido. Isso ocorre pois o Speed utiliza um comitê de classificadores para estimar o nível de congestionamento. Com isso, é possível ter mais acertos na estimativa do tráfego, como destacado na Tabela 2, e consequentemente haverá mais acertos na sugestão de novas rotas. Em relação ao OVMT, nota-se que o Speed é 1.11 vezes mais rápido. Isso está relacionado com o mecanismo de troca de rotas que o OVMT não possui e, por isso, os veículos ficam mais tempo confinados no congestionamento.

Após avaliar o tempo de viagem, analisou-se a emissão de $\mathrm{CO}_{2}$ durante a viagem dos veículos no TMS, como apresentado na Figura 4b. Observa-se que o Speed possui uma redução significativa de $14.17 \%$ na emissão de $\mathrm{CO}_{2}$ quando comparado com o OVMT. Isto faz sentido, pois o OVMT não possui um mecanismo de troca de rotas. Com isso, os veículos que estão na via congestionada demoram mais tempo para finalizar o percurso. Já em relação ao INCIDEnt, nota-se uma redução de 3.73\% na emissão de $\mathrm{CO}_{2}$. Ainda, outro aspecto relevante está relacionado com a baixa dispersão da emissão de $\mathrm{CO}_{2}$ do Speed quando comparado com as soluções implementadas. Isto é identificado pela amplitude interquartil do boxplot. Portanto, o Speed possui uma redução estatisticamente significativa na emissão de $\mathrm{CO}_{2}$. Vale salientar que tal redução pode ser explicada não apenas pelo mecanismo de disseminação de dados que entrega a mensagem em um tempo aceitável, mas também pelo uso do comitê de classificadores que possui uma alta precisão na classificação da via para realizar a alteração da rota.

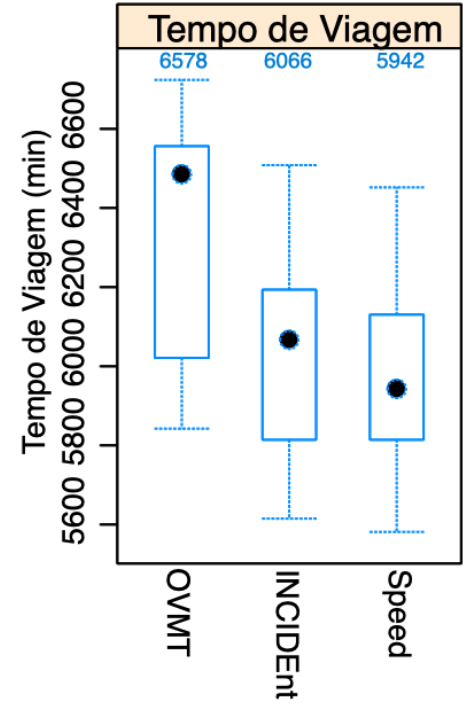

(a) Tempo de viagem

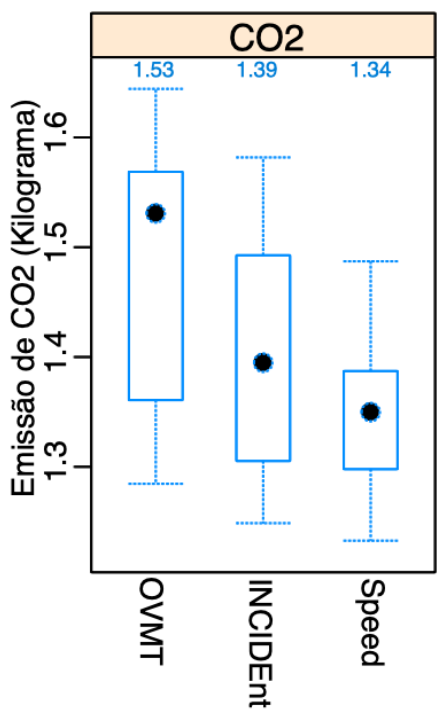

(b) Emissão de $\mathrm{CO}_{2}$

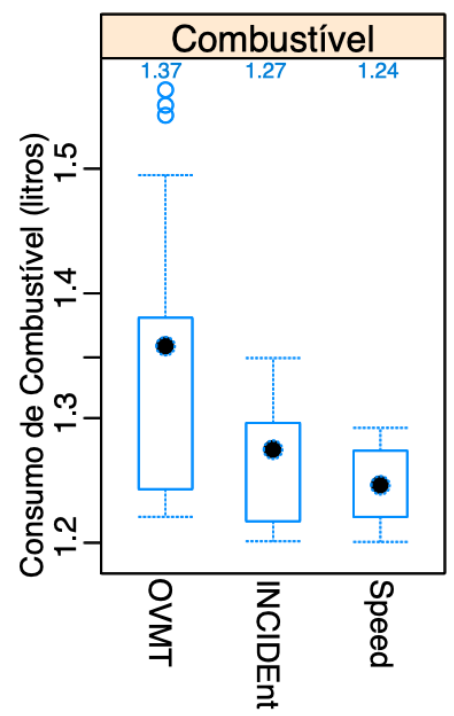

(c) Consumo de combustível

Figura 4. Impacto de desempenho do tempo de viagem, $\mathrm{CO}_{2}$ e Combustível. 
Na Figura 4c, tem-se o objetivo de avaliar a eficiência do Speed no que diz respeito a quantidade de combustível consumida durante o tempo de viagem dos veículos. Observa-se que a quantidade de combustível consumida pelo Speed é ligeiramente menor independente das soluções comparadas (isto é, OVMT e INCIDEnt). Ao comparar com o OVMT e o INCIDEnt, o Speed obteve uma redução de $10.48 \%$ e $3 \%$ no consumo de combustível, respectivamente. Isto ocorre pois, o Speed estima a via com mais precisão e no tempo adequado para sugerir novas rotas. Portanto, o Speed além de reduzir o consumo de combustível e $\mathrm{CO}_{2}$, possui um menor tempo de viagem. Isso é coerente devido a classificação correta do nível de congestionamento da via com um tempo aceitável na disseminação da informação.

\section{Conclusão}

Com o objetivo de atender os problemas encontrados na literatura e superá-los, este artigo propôs o Speed que, além de aumentar a precisão de acertos das vias congestionadas, tem como objetivo detectar o congestionamento da via e maximizar o fluxo de veículos no TMS. Para comprovar a eficiência do Speed, foi realizada uma avaliação de desempenho considerando dois cenários. Os resultados da simulação mostraram que o Speed possui um desempenho superior para todas as métricas avaliadas, quando comparado com outras abordagens da literatura. Salienta-se, portanto, que o Speed evidenciou avanço no estado da arte ao obter uma significativa taxa de acerto para detectar a via congestionada com redução no tempo de viagem, consumo de combustível e emissão de $\mathrm{CO}_{2}$. Como trabalhos futuros, planeja-se além de ampliar o estudo deste trabalho, realizar uma avaliação de desempenho do custo da rede no que diz respeito a disseminação dos dados.

\section{Referências}

Araujo, G., Queiroz, M., Duarte-Figueiredo, F., Tostes, A., and Loureiro, A. (2014). Um protocolo de identificação e minimização de congestionamentos de trafego para redes veiculares. Simpósio Brasileiro de Redes de Computadores e Sistemas Distribuídos-(SBRC).

Bauza, R. and Gozálvez, J. (2013). Traffic congestion detection in large-scale scenarios using vehicle-to-vehicle communications. Journal of Network and Computer Applications, 36(5):1295-1307.

Bauza, R., Gozalvez, J., and Sanchez-Soriano, J. (2010). Road traffic congestion detection through cooperative vehicle-to-vehicle communications. In IEEE Local Computer Network Conference, pages 606-612.

Board, T. R. (2000). Hcm 2000 - highway capacity manual.

Canuto, A. M. d. P. (2001). Combining neural networks and fuzzy logic for applications in character recognition. $\mathrm{PhD}$ thesis, University of Kent at Canterbury.

Cintra, M. (2013). A crise do trânsito em são paulo e seus custos. GVExecutivo, 12(2):5861.

Cunha, F., Maia, G., Ramos, H. S., Perreira, B., Celes, C., Campolina, A., Rettore, P., Guidoni, D., Sumika, F., Villas, L., Mini, R., and Loureiro, A. (2018). Vehicular Networks to Intelligent Transportation Systems, pages 297-315. Springer Singapore, Singapore. 
Doolan, R. and Muntean, G.-M. (2013). Vanet-enabled eco-friendly road characteristicsaware routing for vehicular traffic. In Vehicular Technology Conference (VTC Spring), 2013 IEEE 77th, pages 1-5. IEEE.

Doolan, R. and Muntean, G.-M. (2017). Ecotrec-a novel vanet-based approach to reducing vehicle emissions. IEEE Transactions on Intelligent Transportation Systems, 18(3):608-620.

H. Allen, K. M. and Stonehill, M. (2013). A summary of the proceedings from the united nations climate change conference in doha, qatar, and their significance for the land transport sector, copenhagen: Bridging the gap (btg) initiative.

Junior, G. D., Frozza, R., and Molz, R. F. (2015). Simulação de controle adaptativo de tráfego urbano por meio de sistema multiagentes e com base em dados reais. Revista Brasileira de Computação Aplicada, 7(3):65-81.

Meneguette, R., E. De Grande, R., and A. F. Loureiro, A. (2018). Intelligent Transportation Systems, pages 1-21. Springer International Publishing, Cham.

Meneguette, R. I., Geraldo Filho, P., Guidoni, D. L., Pessin, G., Villas, L. A., and Ueyama, J. (2016). Increasing intelligence in inter-vehicle communications to reduce traffic congestions: experiments in urban and highway environments. PLoS one, 11(8):e0159110.

Meneguette, R. I., Ueyama, J., Krishnamachari, B., Bittencourt, L., et al. (2015). Enhancing intelligence in inter-vehicle communications to detect and reduce congestion in urban centers.

Nottle, A., Quintana-Amate, S., Harborne, D., Alzantot, M., Braines, D., Tomsett, R., Kaplan, L., Srivastava, M., Chakraborty, S., and Preece, A. (2017). Distributed opportunistic sensing and fusion for traffic congestion detection. In First International Workshop on Distributed Analytics InfraStructure and Algorithms for Multi-Organization Federations, pages 1-6.

Pan, J. S., Khan, M. A., Popa, I. S., Zeitouni, K., and Borcea, C. (2012). Proactive vehicle re-routing strategies for congestion avoidance. In 2012 8th IEEE International Conference on Distributed Computing in Sensor Systems, pages 265-272. IEEE.

Pan, J. S., Popa, I. S., and Borcea, C. (2017). Divert: A distributed vehicular traffic rerouting system for congestion avoidance. IEEE Transactions on Mobile Computing, 16(1):58-72.

Pessin, G., Souza, J. R., Osório, F. S., Faiçal, B. S., Geraldo Filho, P., Ueyama, J., Vargas, P. A., and Wolf, D. F. (2014). Investigation on the evolution of a robotic controller for autonomous vehicle navigation. Journal of Intelligent \& Fuzzy Systems, 27(6):30473058 .

Rao, A. M. and Rao, K. R. (2012). Measuring urban traffic congestion-a review. International Journal for Traffic \& Transport Engineering, 2(4).

Santos, A. d. M. (2008). Uma análise da aplicação do modelo de rede neural repart em comitês de classificadores. Master's thesis, Universidade Federal do Rio Grande do Norte. 
Severino, J. N., Cristiani, A. L., Nakamura, L. H., and Meneguette, R. I. (2018). Urbtrans: A system for mobility management of school vans for cities in the interior. In Proceedings of the 10th Latin America Networking Conference, pages 10-17. ACM.

SKYCOMP (2008). Major highway performance ratings and bottleneck inventory. Visitado em Outubro de 2018, http: / / www. skycomp. com/MDSHA/resources / Spring_2008.pdf.

Souza, A. M., Brennand, C. A., Yokoyama, R. S., Donato, E. A., Madeira, E. R., and Villas, L. A. (2017). Traffic management systems: A classification, review, challenges, and future perspectives. International Journal of Distributed Sensor Networks, 13(4):1550147716683612.

Souza, A. M., Guidoni, D., Botega, L. C., and Villas, L. A. (2015). Co-op: Uma solução para a detecção, classificação e minimização de congestionamentos de veículos utilizando roteamento cooperativo. Simpósio Brasileiro de Redes de Computadores e Sistemas Distribuídos-(SBRC).

Texas Transportation Institute, D.L. Schrank, W. T. J. (2015). 2015 urban mobility scorecard, mobility report.

Wongcharoen, S. and Senivongse, T. (2016). Twitter analysis of road traffic congestion severity estimation. In Computer Science and Software Engineering (JCSSE), 2016 13th International Joint Conference on, pages 1-6. IEEE. 